\title{
Lepton flavor violating tau decays in the presence of massive neutrinos
}

\author{
G. Hernández-Tomé* \\ CAFPE and Departamento de Física Teórica y del Cosmos, Universidad de Granada, E-18071 \\ Granada, Spain \\ E-mail: ghernandezt@correo.ugr.es
}

\begin{abstract}
We discuss the lepton flavor violating decays of a $\tau$ lepton into three lighter ones charged leptons in the presence of heavy Majorana neutrinos. Updated predictions obeying the current limits of the heavy-light mixing angles are presented. We point out the differences between the case of two non-degenerate and degenerate heavy Majorana neutrinos. In the latter, the two heavy states reduce to a single Dirac neutrino, where the lepton number violating effects vanish.
\end{abstract}

7th Annual Conference on Large Hadron Collider Physics - LHCP2019

20-25 May, 2019

Puebla, Mexico

${ }^{*}$ Speaker. 


\section{Introduction}

As is well known, the observation of neutrino oscillations provides undeniable evidence of lepton flavor violation (LFV) in the neutrino sector, and claims for an extension of the electroweak model of Glashow-Weinberg-Salam that incorporates massive neutrinos. A possible extension is the so-called $v \mathrm{SM}[1]$, where singlet right-handed neutrino fields are introduced in the Yukawa Lagrangian; then the neutrino masses are generated via couplings with the Higgs doublet, just as it occurs for all the other fermions. In the $v \mathrm{SM}$, the mixing in the leptonic sector is described by a $3 \times 3$ unitary matrix called the PMNS matrix [2,3], analogous to the CKM matrix of the quark sector $[4,5]$. However, the $v$ SM requires extremely tiny Yukawa couplings to explain the observed masses of the neutrinos, which suggests that another mechanism may be at work. If, in addition to the Dirac mass terms $\left(m_{D}\right)$ that combine them with the active neutrinos, the singlets have Majorana masses $\left(m_{M}\right)$ that define a new scale ${ }^{1}$, then the tiny neutrino masses may appear naturally for a very large value of $m_{M}$ (i.e., $m_{M} \gg m_{D}$ ). In this latter scenario known as type-I seesaw $[6,7,8]$, the physical states after diagonalization of the mass matrix includes light $(v)$ and heavy Majorana neutrinos $(N)$ with masses $m_{v} \approx m_{D}^{2} / m_{M}$, and $m_{N} \approx m_{M} \gg m_{v}$, respectively. In the case of only one generation of heavy neutrinos, the heavy-light mixings are fixed by the relation

$$
s_{v} \approx m_{D} / m_{M} \approx \sqrt{m_{v} / m_{N}} .
$$

In the presence of massive neutrinos, charged lepton flavor violating (cLFV) transitions like $\ell \rightarrow$ $\ell^{\prime} \gamma$, and $\ell \rightarrow \ell^{\prime} \ell^{\prime \prime} \bar{\ell}^{\prime \prime \prime}$, where $\ell, \ell^{\prime}, \ell^{\prime \prime}$ and $\ell^{\prime \prime \prime}$ denote usual charged leptons $(\tau, \mu, e)$, can emerge at one loop level mediated by the weak charged current. Nevertheless, these effects turn out strongly suppressed both in the $v \mathrm{SM}$ and in the usual type-I seesaw model. In the former, the suppression comes from a GIM-like mechanism where the amplitudes are proportional to squared mass differences of the light neutrinos (the branching ratios are then below $10^{-50}[9,10,11]$ ). In the latter, the heavy neutrino effects are irrelevant because of the relation (1.1) (the amplitudes are proportional to the square mass of the heavy neutrinos but are suppressed by a product of heavy-light mixing angles). However, there are some well-motivated variants of the usual type-I seesaw scenario predicting new heavy neutrino states, whose mixings with the light neutrinos are unsuppressed, but constrained only by the experimental limits. These models, which require at least two heavy Majorana neutrinos, are justified by approximate symmetries or some ansatz on the neutrino mass matrix that relaxes the restriction of Eq. (1.1). Examples of these models are the inverse seesaw $[12,13]$ and the linear seesaw [14]. In these models, significant rates for cLFV transitions might occur.

In this work, we study the lepton flavor violating decays of a $\tau$ lepton into three lighter charged leptons $\left(\tau \rightarrow \ell^{\prime} \ell^{\prime \prime} \bar{\ell}^{\prime \prime \prime}\right)$ in the presence of heavy Majorana neutrinos. The current and future sensitivities for these transitions are shown in Table 1. In section 2, we present the relevant Lagrangian densities involved in our computation. Section 3 presents analytic expressions of all the possible form factors associated with the different Feynman diagram contributions. A numerical evaluation of our results, obeying the current limits of the heavy-light mixing angles, as well as

\footnotetext{
${ }^{1}$ A Majorana mass term for a singlet right-handed neutrino is not forbidden by the gauge symmetry. This term breaks explicitly the accidental lepton number symmetry of the SM since it involves mixing between a neutrino and its charged conjugate partner (antineutrino) with opposite fermion number.
} 


\begin{tabular}{|c|c|}
\hline Reaction & Present Limit 90\% C.L \\
\hline$\tau \rightarrow e e \bar{e}$ & $2.7 \times 10^{-8}$ \\
\hline$\tau \rightarrow \mu \mu \bar{\mu}$ & $2.1 \times 10^{-8}$ \\
\hline$\tau \rightarrow \mu e \bar{e}$ & $1.8 \times 10^{-8}$ \\
\hline$\tau \rightarrow e \mu \bar{\mu}$ & $2.7 \times 10^{-8}$ \\
\hline$\tau \rightarrow \mu \mu \bar{e}$ & $1.7 \times 10^{-8}$ \\
\hline$\tau \rightarrow e e \bar{\mu}$ & $1.5 \times 10^{-8}$ \\
\hline
\end{tabular}

Table 1: Present limits from Belle [15] for $\tau \rightarrow \ell^{\prime} \ell^{\prime \prime} \bar{\ell}^{\prime \prime \prime}$ cLFV processes. Future sensitivities are $\sim 2-5 \times$ $10^{-10}$ [16]. LHCb has already set a limit of $4.6 \times 10^{-8}$ [17] for the $\tau \rightarrow \mu \mu \bar{\mu}$ decay, very competitive with the present limit of Belle. In its high-luminosity phase, the LHC is expected to improve this bound by one order of magnitude.

a discussion of the differences between the case of two non-degenerate and degenerate heavy Majorana neutrinos is presented in section 4. Finally, section 5 is devoted to our conclusions.

\section{Theoretical framework}

We work in an interfamily model with a pair of extra heavy Majorana singlets and arbitrary mixings with the three active neutrino flavors. A complete description of the model can be found in $[18,19,20]$. The charged and neutral currents, in terms of physical neutrinos $\chi_{i}$, are given by:

$$
\begin{aligned}
\mathscr{L}_{W^{ \pm}} & =-\frac{g}{\sqrt{2}} W_{\mu}^{-} \sum_{i=1}^{3} \sum_{j=1}^{5} B_{i j} \bar{\ell}_{i} \gamma^{\mu} P_{L} \chi_{j}+\text { h.c.. } \\
\mathscr{L}_{Z} & =-\frac{g}{4 c_{W}} Z_{\mu} \sum_{i, j=1}^{5} \bar{\chi}_{i} \gamma^{\mu}\left(C_{i j} P_{L}-C_{i j}^{*} P_{R}\right) \chi_{j}, \\
\mathscr{L}_{G^{ \pm}} & =-\frac{g}{\sqrt{2} m_{W}} G^{-} \sum_{i=1}^{3} \sum_{j=1}^{5} B_{i j} \bar{\ell}_{i}\left(m_{\ell_{i}} P_{L}-m_{\chi_{j}} P_{R}\right) \chi_{j}+\text { h.c. },
\end{aligned}
$$

where $G^{ \pm}$is the charged would-be-Goldstone field, $g$ is the weak coupling constant, $c_{W}=\cos \theta_{W}$ and $P_{L, R}=\frac{1}{2}\left(1 \mp \gamma_{5}\right)$ are the left and right-handed projectors, respectively. Notice that in Eq. (2.2), the neutral current induced by the Majorana states involves couplings of different flavors with both left and right-handed components. ${ }^{2}$ The first three states $\chi_{1,2,3}$, identified with the three (mostly) active neutrinos are exactly massless, whereas the masses of the two heavy states are $m_{\chi_{4}}=m_{N_{1}}$ and $m_{\chi_{5}}=m_{N_{2}}$.

The dimension of the rectangular $B$ mixing matrix is $3 \times 5$, whereas $C$ is a $5 \times 5$ matrix. The elements of these matrices involving heavy neutrinos can be expressed in terms of heavy-light mixings and the squared mass ratio $r=m_{N_{2}}^{2} / m_{N_{1}}^{2}$ by

$$
B_{k N_{1}}=-\frac{i r^{\frac{1}{4}}}{\sqrt{1+r^{\frac{1}{2}}}} s_{v_{k}}, \quad B_{k N_{2}}=\frac{1}{\sqrt{1+r^{\frac{1}{2}}}} s_{v_{k}},
$$

\footnotetext{
${ }^{2}$ For the case of heavy left-handed neutrinos being sequential Dirac (active) neutrinos, replace $B_{i j} \rightarrow U_{i j}^{v}, C_{i j} \rightarrow \delta_{i j}$, $C_{i j}^{*} \rightarrow 0$.
} 


$$
C_{N_{1} N_{1}}=\frac{r^{\frac{1}{2}}}{1+r^{\frac{1}{2}}} \sum_{k=1}^{3} s_{v_{k}}^{2}, \quad C_{N_{2} N_{2}}=\frac{1}{1+r^{\frac{1}{2}}} \sum_{k=1}^{3} s_{v_{k}}^{2}, C_{N_{1} N_{2}}=-C_{N_{2} N_{1}}=\frac{i r^{\frac{1}{4}}}{1+r^{\frac{1}{2}}} \sum_{k=1}^{3} s_{v_{k}}^{2} .
$$

The above expressions are the same as those reported in $[18,21]$ up to an irrelevant global phase for $B$. Furthermore, the matrices $B$ and $C$ satisfy some identities that are essential to keep the renormalizability of the model:

$$
\begin{aligned}
\sum_{k=1}^{5} B_{i k} B_{j k}^{*} & =\delta_{i j}, \quad \sum_{k=1}^{3} B_{k i}^{*} B_{k j}=\sum_{k=1}^{5} C_{i k} C_{j k}^{*}=C_{i j}, \quad \sum_{k=1}^{5} B_{i k} C_{k j}=B_{i j}, \\
\sum_{k=1}^{5} m_{\chi_{k}} C_{i k} C_{j k} & =\sum_{k=1}^{5} m_{\chi_{k}} B_{i k} C_{k j}^{*}=\sum_{k=1}^{5} m_{\chi_{k}} B_{i k} B_{j k}=0 .
\end{aligned}
$$

3. $\ell \rightarrow \ell^{\prime} \ell^{\prime \prime} \bar{\ell}^{\prime \prime \prime}$ decays
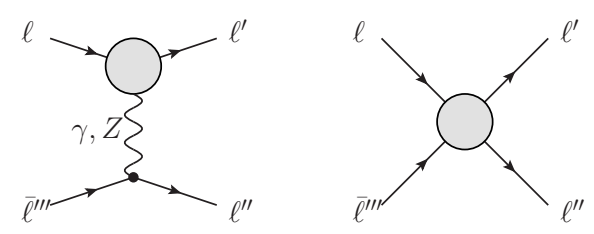

Figure 1: Generic penguin and box diagrams contributing to $\ell \rightarrow \ell^{\prime} \ell^{\prime \prime} \ell^{\prime \prime \prime}$. The gray circle in the diagram on the left represents the effective $V \ell \ell^{\prime}(V=\gamma, Z)$ vertex, which is generated by the diagrams in Fig. 2, whereas the gray circle in the other diagram stands for all the possible box contributions given in Fig. 3.
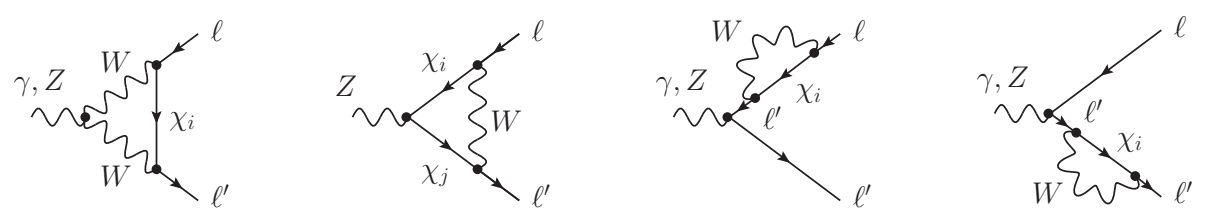

Figure 2: One-loop diagrams contributing to the $V \ell \ell^{\prime}$ vertex. From left to right, the second diagram only contributes to the $Z \ell \ell^{\prime}$ vertex since the photon does not interact directly with neutrinos. We have worked in the Feynman-'t Hooft guage, where additional diagrams with the $W$ gauge boson replace by the respective would-be Goldstone boson must be taking into account.

We now present the amplitudes for the $\ell \rightarrow \ell^{\prime} \ell^{\prime \prime} \ell^{\prime \prime \prime}$ decays. They can be split into three types of decays according to Table 2 . In general, these processes have generic photon-penguin, $Z$-penguin and box contributions, such as is depicted in Fig. 1. The total amplitude is then given by

$$
\mathscr{M}\left(\ell \rightarrow \ell^{\prime} \ell^{\prime \prime} \bar{\ell}^{\prime \prime \prime}\right)=\mathscr{M}_{\gamma}+\mathscr{M}_{Z}+\mathscr{M}_{B o x}
$$



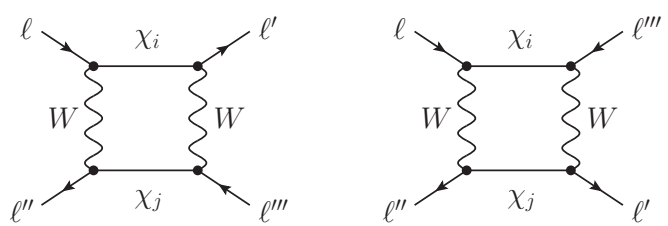

Figure 3: Box diagrams contributing to $\ell \rightarrow \ell^{\prime} \ell^{\prime \prime} \bar{\ell}^{\prime \prime \prime}$. Note that the diagram on the right introduces explicit LNV vertices. When masses and momenta of the external particles are neglected in the computation all the box contributions can be grouped in terms of just one form factor associated with a single Lorentz structure.

\begin{tabular}{|c|l|}
\hline & $\ell \rightarrow \ell^{\prime} \ell^{\prime \prime} \bar{\ell}^{\prime \prime \prime}$ \\
\hline \multirow{3}{*}{ Type $1\left(\ell^{\prime}=\ell^{\prime \prime}\right.$ and $\left.\ell^{\prime \prime}=\ell^{\prime \prime \prime}\right)$} & $\mu \rightarrow e e \bar{e}$ \\
& $\tau \rightarrow \mu \mu \bar{\mu}$ \\
& $\tau \rightarrow e e \bar{e}$ \\
\hline Type 2 $\left(\ell^{\prime} \neq \ell^{\prime \prime}\right.$ and $\left.\ell^{\prime \prime}=\ell^{\prime \prime \prime}\right)$ & $\tau \rightarrow \mu e \bar{e}$ \\
& $\tau \rightarrow e \mu \bar{\mu}$ \\
\hline Type 3 $\left(\ell^{\prime}=\ell^{\prime \prime}\right.$ and $\left.\ell^{\prime \prime} \neq \ell^{\prime \prime \prime}\right)$ & $\tau \rightarrow \mu \mu \bar{e}$ \\
& $\tau \rightarrow e e \bar{\mu}$ \\
\hline
\end{tabular}

Table 2: Possible $\ell \rightarrow \ell^{\prime} \ell^{\prime \prime} \bar{\ell}^{\prime \prime \prime}$ channels. Besides of the $\mu \rightarrow e e \bar{e}$ decay, the $\tau$ lepton has six different decay channels. Type 1 and Type 2 channels receive generic contributions from $\gamma$-penguin, $Z$-penguin, and box diagrams. Type 3 channels only have box contributions, as they require two flavor changing vertices.

the individual contributions are determined by

$$
\begin{aligned}
\mathscr{M}_{\gamma} & =\frac{e^{2}}{q^{2}}\left[\bar{u}\left(p_{\ell^{\prime}}\right)\left(F_{L}^{\gamma} \gamma_{\mu} P_{L}+i F_{M}^{\gamma}\left(1+\gamma_{5}\right) \sigma_{\mu v} q^{v}\right) u\left(p_{\ell}\right)\right]\left[\bar{u}\left(p_{\ell^{\prime \prime}}\right) \gamma^{\mu} v\left(p_{\ell^{\prime \prime \prime}}\right)\right]-\left(\ell^{\prime} \leftrightarrow \ell^{\prime \prime}\right), \\
\mathscr{M}_{Z} & =-\frac{e^{2}}{m_{Z}^{2}} F_{L}^{Z}\left[\bar{u}\left(p_{\ell^{\prime}}\right) \gamma_{\mu} P_{L} u\left(p_{\ell}\right)\right] \times\left[\bar{u}\left(p_{\ell^{\prime \prime}}\right) \gamma^{\mu}\left(g_{L}^{Z} P_{L}+g_{R}^{Z} P_{R}\right) v\left(p_{\ell^{\prime \prime \prime}}\right)\right]-\left(\ell^{\prime} \leftrightarrow \ell^{\prime \prime}\right), \\
\mathscr{M}_{B o x} & =e^{2} F_{B}\left[\bar{u}\left(p_{\ell^{\prime}}\right) \gamma_{\mu} P_{L} u\left(p_{\ell}\right)\right]\left[\bar{u}\left(p_{\ell^{\prime \prime}}\right) \gamma^{\mu} P_{L} v\left(p_{\ell^{\prime \prime \prime}}\right)\right],
\end{aligned}
$$

where $q^{v}$ is the momentum transferred by the $V$ boson, and $g_{L}^{Z}=\frac{g}{2 c_{W}}\left(-1+2 s_{W}^{2}\right), g_{R}^{Z}=\frac{g}{c_{W}} s_{W}^{2}$ are the left and right couplings of a charged lepton pair with the $Z$ gauge boson, respectively. Note that, in Eqs. (3.2) and (3.3), the crossed diagrams ( $\ell^{\prime} \leftrightarrow \ell^{\prime \prime}$ terms) that antisymmetrize the amplitudes only apply for the type 1 and type 3 channels in Table 2. Besides, we have included in the form factor $F_{B}$ of Eq. (3.4) the crossed box diagram using the Fierz identity

$$
\left\langle a\left|\gamma^{\mu} P_{L}\right| b\right\rangle\left\langle c\left|\gamma_{\mu} P_{L}\right| d\right\rangle=-\left\langle c\left|\gamma^{\mu} P_{L}\right| b\right\rangle\left\langle a\left|\gamma_{\mu} P_{L}\right| d\right\rangle
$$

The partial widths for the different types are presented in the appendix A. 
The relevant form factors involved in the above amplitudes are then given by

$$
\begin{aligned}
F_{L}^{\gamma} & =\frac{\alpha_{W}}{8 \pi m_{W}^{2}} \sum_{i}^{5} B_{\ell i}^{*} B_{\ell^{\prime}} f_{L}^{\gamma}\left(x_{i} ; q^{2}\right), \\
F_{M}^{\gamma} & =\frac{\alpha_{W}}{8 \pi m_{W}^{2}} \frac{m_{\ell}}{2} \sum_{i}^{5} B_{\ell i}^{*} B_{\ell^{\prime} i} f_{M}^{\gamma}\left(x_{i}\right), \\
F_{L}^{Z} & =\frac{\alpha_{W}}{8 \pi s_{W} c_{W}} \sum_{i, j}^{5} B_{\ell i}^{*} B_{\ell^{\prime} j}\left[F\left(x_{i}\right) \delta_{i j}+C_{i j}^{*} G\left(x_{i}, x_{j}\right)+C_{i j} \sqrt{x_{i} x_{j}} H\left(x_{i}, x_{j}\right)\right] \\
F_{B} & =\frac{\alpha_{W}}{16 \pi m_{W}^{2} s_{W}^{2}} \sum_{i, j}^{5}\left\{B_{\ell i}^{*} B_{\ell^{\prime \prime \prime} j}^{*}\left[B_{\ell^{\prime} i} B_{\ell^{\prime \prime} j}+B_{\ell^{\prime \prime} i} B_{\ell^{\prime} j}\right] f_{B}^{L N C}\left(x_{i}, x_{j}\right)+B_{\ell i}^{*} B_{\ell^{\prime \prime} i}^{*} B_{\ell^{\prime} j} B_{\ell^{\prime \prime} j} f_{B}^{L N V}\left(x_{i}, x_{j}\right)\right\},
\end{aligned}
$$

where we defined $x_{i} \equiv \frac{m_{\chi_{i}}^{2}}{m_{W}^{2}}$. All the form factors can be expressed in terms of only the heavy states [19]. The loop functions associated with the effective $\gamma \ell \ell^{\prime}$ vertex are given by

$$
\begin{aligned}
f_{L}^{\gamma}\left(x ; q^{2}\right) & =\left[\frac{x^{2}\left(x^{2}-10 x+12\right) \log x}{6(x-1)^{4}}+\frac{\left(7 x^{3}-x^{2}-12 x\right)}{12(x-1)^{3}}-\frac{5}{9}\right] q^{2}+2 m_{W}^{2} \Delta_{\varepsilon} \\
f_{M}^{\gamma}(x) & =\frac{3 x^{3} \log x}{2(x-1)^{4}}-\frac{2 x^{3}+5 x^{2}-x}{4(x-1)^{3}}+\frac{5}{6}
\end{aligned}
$$

where $\Delta_{\varepsilon}=\frac{1}{\varepsilon}-\gamma_{E}+\log (4 \pi)+\log \left(\frac{\mu^{2}}{m_{W}^{2}}\right)$ is an ultraviolet divergence. Concerning the functions associated with the $Z$-penguin contributions, we have

$$
\begin{aligned}
F(x) & =\frac{5 x^{2} \log x}{2(x-1)^{2}}-\frac{5 x}{2(x-1)}+\frac{1}{4}-\left(\frac{5}{2}-2 s_{W}^{2}\right) \Delta_{\varepsilon}, \\
G(x, y) & =\frac{1}{2(x-y)}\left[\frac{x^{2}(y-1) \log x}{(x-1)}-\frac{(x-1) y^{2} \log y}{(y-1)}\right]+\frac{1}{2}\left(\Delta_{\varepsilon}-\frac{1}{2}\right), \\
H(x, y) & =\frac{1}{4(x-y)}\left(\frac{(x-4) x \log x}{x-1}-\frac{(y-4) y \log y}{y-1}\right)-\frac{1}{4}\left(\Delta_{\varepsilon}+\frac{1}{2}\right) .
\end{aligned}
$$

All ultraviolet divergences above cancel in the form factors using:

$$
\sum_{i, j}^{5} B_{\ell i}^{*} B_{\ell^{\prime} i}=0, \quad \sum_{i, j}^{5} B_{\ell i}^{*} B_{\ell^{\prime} j} C_{i j}^{*}=\sum_{j}^{5} B_{\ell j}^{*} B_{\ell^{\prime} j}=0, \quad \sum_{i}^{5} m_{\chi_{i}} B_{\ell i}^{*} C_{i j}=0 .
$$

Finally, the loop functions associated with the box contributions are

$$
\begin{aligned}
& f_{B}^{L N C}(x, y)=\left(1+\frac{x y}{4}\right) \tilde{d}_{0}(x, y)-2 x y d_{0}(x, y), \\
& f_{B}^{L N V}(x, y)=\sqrt{x y}\left[2 \tilde{d}_{0}(x, y)-(4+x y) d_{0}(x, y)\right],
\end{aligned}
$$

where

$$
\begin{aligned}
& \tilde{d}_{0}(x, y)=-\frac{1}{(x-1)(y-1)}-\frac{x^{2} \log (x)}{(x-1)^{2}(x-y)}+\frac{y^{2} \log (y)}{(y-1)^{2}(x-y)} \\
& d_{0}(x, y)=-\frac{1}{(x-1)(y-1)}-\frac{x \log (x)}{(x-1)^{2}(x-y)}+\frac{y \log (y)}{(y-1)^{2}(x-y)}
\end{aligned}
$$


It is important to highlight that the amplitude in (3.4), includes the contribution of Feynman diagrams with explicit LNV vertices. We have followed the algorithm implemented by Denner [22], which circumvents the explicit introduction of the charged conjugation matrix in the Feynman rules and allows to use the standard propagators for Majorana particles. We emphasize that the box diagrams on the right in Fig. 3 are genuine contributions from Majorana particles that should vanish in models with conserved lepton number. We have verified that this happens when the two fermion singlets define a Dirac field. We have also checked that our expressions agree with those in [18].

\section{Numerical Analysis}

We now evaluate the predictions for the $\tau \rightarrow \ell^{\prime} \ell^{\prime \prime} \bar{\ell}^{\prime \prime \prime}$ decays in the theoretical framework presented in section 2. In this scenario, there are only five free parameters: the heavy neutrinos masses $m_{N_{1}}$ and $m_{N_{2}}$ (or equivalently $m_{N_{1}}$ and $r$ ), and the three heavy-light mixing angles $s_{v_{e}}$, $s_{v_{\mu}}$ and $s_{v_{\tau}}$. Note that, even though neutrino masses and mixing angles can be considered free parameters in this model, their values must maintain the validity of perturbative unitarity to have a realistic scenario. Therefore, if we assume not very small values of the mixing angles, neutrinos masses can not be arbitrarily heavy. The perturbative unitarity condition can be ensured qualitatively provided that Yukawa couplings satisfy the relation

$$
Y_{N_{\alpha}}=\frac{\sqrt{2 m_{N_{1}} m_{N_{2}}}}{v} s_{v_{\alpha}}<\sqrt{4 \pi}
$$

Furthermore, we will only consider mixings that respect the indirect limits given in [23]:

$$
s_{v_{e}}<0.050(0.031), \quad s_{v_{\mu}}<0.021(0.011), \quad s_{v_{\tau}}<0.075(0.044) .
$$

These limits were obtained at the $2 \sigma(1 \sigma)$ level performing a global fit to a set of electroweak precision data and lepton flavor conserving processes with the effects of extra neutrinos encoded in effective operators. They are independent from the heavy neutrino masses.

On the other hand, we also consider the direct limit from the $\mu \rightarrow e \gamma$ decay, that for heavy neutrinos (above $\sim 1 \mathrm{TeV}$ ) comes from [21]

$$
\mathrm{BR}(\mu \rightarrow e \gamma) \approx \frac{3 \alpha}{8 \pi} s_{v_{e}}^{2} s_{v_{\mu}}^{2}
$$

which yields

$$
s_{v_{e}}^{2} s_{v_{\mu}}^{2}<4.82 \times 10^{-10} .
$$

Fig. 4 shows the branching ratio for the $\tau \rightarrow \ell^{\prime} \ell^{\prime \prime} \bar{\ell}^{\prime \prime \prime}$ decays as a function of the neutrino mass for the case $m_{N}=m_{N_{1}}=m_{N_{2}}$. Several important comments are in order:

- We consider the optimistic scenario, where the heavy-light mixing angles take the maximum values allowed by the indirect constraints at $2 \sigma(1 \sigma)$ level and the direct limit in Eq. (4.4).

- We focus on the $\tau \rightarrow e e \bar{e}, \tau \rightarrow e \mu \bar{\mu}$, and $\tau \rightarrow e e \bar{\mu}$ decays. A similar analysis can be done for the channels with $e \leftrightarrow \mu$ if we interchange the maximal indirect value of $s_{v_{e}}$ by that of $s_{v_{\mu}}$ from Eq. (4.2) in Eq. (4.4). 


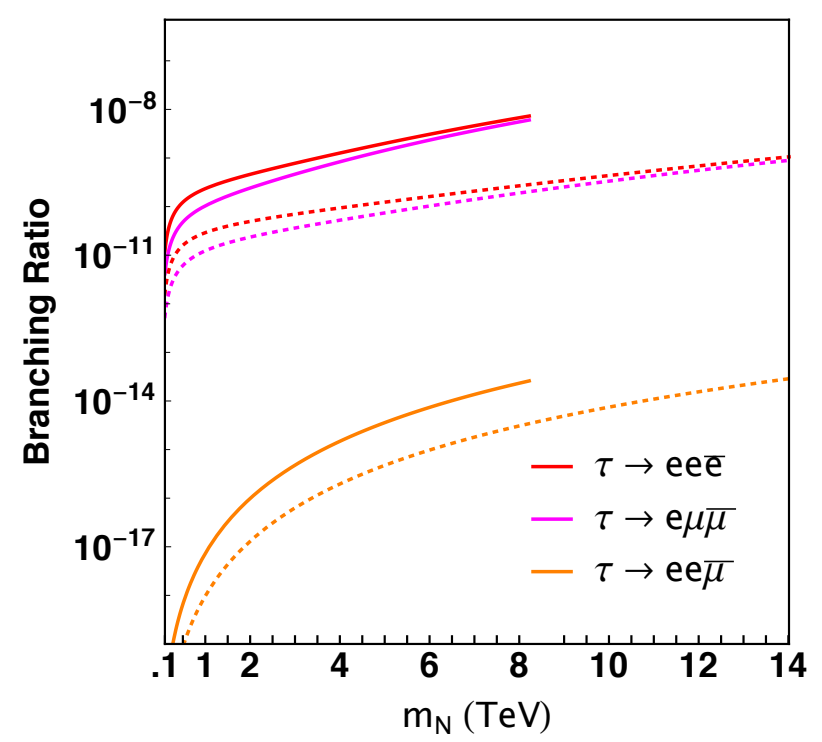

Figure 4: Branching ratios for $\tau \rightarrow \ell^{\prime} \ell^{\prime \prime} \bar{\ell}^{\prime \prime \prime}$ decays considering $m_{N}=m_{N_{1}}=m_{N_{2}}$. The solid (dashed) lines stand for the maximum values of the heavy-light mixing obeying the direct limit in Eq. (4.4) and the indirect ones in Eq. (4.2) at $2 \sigma(1 \sigma)$ level. We only consider values of $m_{N}$ allowed by the perturbative unitarity condition.

- The predictions for $\tau \rightarrow e e \bar{e}$ and $\tau \rightarrow e \mu \bar{\mu}$ are very close. This is due to the fact that the $Z$ penguin contribution, involving the effective $Z \tau e$ vertex, dominates in both channels.

- The $\tau \rightarrow e e \bar{e}$ and $\tau \rightarrow e \mu \bar{\mu}$ decays are promising, since taking the maximum value for $m_{N}$ allowed by Eq. (4.1), we have that

$$
\begin{aligned}
\operatorname{BR}(\tau \rightarrow e e \bar{e}) & \sim 7.29 \times 10^{-9}\left(1.05 \times 10^{-9}\right), \\
\operatorname{BR}(\tau \rightarrow e \mu \bar{\mu}) & \sim 6.04 \times 10^{-9}\left(8.94 \times 10^{-10}\right),
\end{aligned}
$$

which would be at reach of experiments like Belle-2 [16].

- Since the $\tau \rightarrow e e \bar{\mu}$ is generated only by box diagrams its amplitude is always proportional to $s_{v_{e}}^{2} s_{v_{\tau}} s_{v_{\mu}}$. Then it is suppressed in comparison with the other channels:

$$
\operatorname{BR}(\tau \rightarrow e e \bar{\mu}) \sim 2.59 \times 10^{-14}\left(2.80 \times 10^{-14}\right) .
$$

The analysis above was for the case of two mass-degenerate heavy Majorana neutrinos, which combine to form a heavy singlet Dirac. In order to illustrate now the effects due to the mass-splitting of the two heavy neutral states, Fig. 5 shows the ratio $\operatorname{BR}\left(\tau \rightarrow \ell^{\prime} \ell^{\prime} \bar{\ell}^{\prime \prime \prime}\right)(r) / \operatorname{BR}\left(\tau \rightarrow \ell^{\prime} \ell^{\prime} \bar{\ell}^{\prime \prime \prime}\right)(r=1)$ as a function of $r$, for two fixed values of $m_{N_{1}}$ and maximal heavy-light mixing angles. We can see that the predictions for the $\tau \rightarrow e e \bar{e}$ and $\tau \rightarrow e \mu \bar{\mu}$ decays change by a small relative factor. For example, given $m_{N_{1}}=3 \mathrm{TeV}$ and the maximum values for the heavy-light mixings angles at $2 \sigma$ level, the prediction for the $\tau \rightarrow e \mu \bar{\mu}$ is enhanced by a factor $\sim 2$. However, the $\tau \rightarrow$ $e e \bar{\mu}$ decay is very sensitive to the genuine effects of Majorana neutrinos, coming from the LNV 


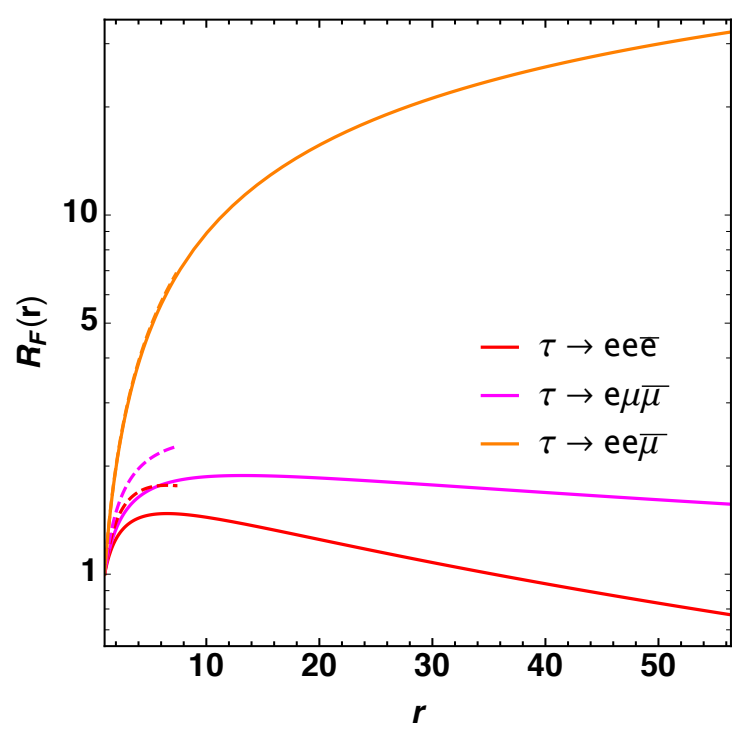

Figure 5: Effects due to the mass-splitting $\left(r \equiv m_{N_{2}}^{2} / m_{N_{1}}^{2}\right.$ ) of two heavy Majorana neutrinos. The solid line shows the ratios $R_{F}(r)=\operatorname{BR}\left(\tau \rightarrow \ell^{\prime} \ell^{\prime} \bar{\ell}^{\prime \prime \prime}\right)(r) / \mathrm{BR}\left(\tau \rightarrow \ell^{\prime} \ell^{\prime} \bar{\ell}^{\prime \prime \prime}\right)(r=1)$ for $m_{N_{1}}=3 \mathrm{TeV}$ with maximum values of the heavy-light mixing allowed by Eq. (4.4) and Eq. (4.2) at $2 \sigma$ level. The dashed line is for $m_{N_{1}}=5 \mathrm{TeV}$. Only points compatible with perturbative unitarity are plotted.

box contributions. Unlike the $\tau \rightarrow e e \bar{e}$ and $\tau \rightarrow e \mu \bar{\mu}$ decays, which are dominated by the $Z$ penguin contributions, in the $\tau \rightarrow e e \bar{\mu}$ transition, the relative factor between the non-degenerate and degenerate cases keeps growing if the splitting on masses also does. Then the observation of one of these decays could suggest the presence of LNV effects.

\section{Conclusions}

The nature of neutrinos is still an unanswered question: we do not know whether neutrinos are Dirac or Majorana particles. Models with a non-minimal neutrino sector open a window to new physics effects. In particular, we have seen that some scenarios may incorporate extra heavy neutrinos with unsuppressed heavy-light mixings, which can give origin to cLFV transitions at measurable rates. In this work, we have studied the $\tau \rightarrow \ell^{\prime} \ell^{\prime \prime} \bar{\ell}^{\prime \prime \prime}$ decays in the simplest model that captures these features. Analytic expressions for all the form factors of the different Feynman diagram topologies are presented. Our results complete and update previous results by other authors $[18,24,25]$.

\section{Acknowledgments}

I would like to thank the organizers of LHCP2019 for the given time. I also thank J. I. Illana, G. López, M. Masip and P. Roig for the collaboration. The presentation of this talk would not have been possible without the funding of CONACYT through the program "estancia postdoctoral en el extranjero". 


\section{A. Partial widths}

After integrating over the three-body phase space one obtains the decay widths

$$
\begin{aligned}
\Gamma\left(\ell \rightarrow \ell^{\prime} \ell^{\prime \prime} \bar{\ell}^{\prime \prime \prime}\right)_{\text {Type } 1} & =\frac{\alpha^{2} m_{\ell}^{5}}{96 \pi}\left\{3\left|A_{1 L}\right|^{2}+2\left|A_{2 R}\right|^{2}\left(8 \log \left(\frac{m_{\ell}}{m_{\ell^{\prime \prime}}}\right)-13\right)+2\left|F_{L L}\right|^{2}+\left|F_{L R}\right|^{2}+\frac{1}{2}\left|F_{B}\right|^{2}\right. \\
& \left.-\left[6 A_{1 L} A_{2 R}{ }^{*}-\left(A_{1 L}-2 A_{2 R}\right)\left(2 F_{L L}{ }^{*}+F_{L R}{ }^{*}+F_{B}{ }^{*}\right)-F_{L L} F_{B}{ }^{*}+\text { h.c. }\right]\right\}, \quad \text { (A.1) } \\
\Gamma\left(\ell \rightarrow \ell^{\prime} \ell^{\prime \prime} \bar{\ell}^{\prime \prime \prime}\right)_{\text {Type } 2} & =\frac{\alpha^{2} m_{\ell}^{5}}{96 \pi}\left\{2\left|A_{1 L}\right|^{2}+4\left|A_{2 R}\right|^{2}\left(4 \log \left(\frac{m_{\ell}}{m_{\ell^{\prime \prime}}}\right)-7\right)+\left|F_{L L}\right|^{2}+\left|F_{L R}\right|^{2}+\left|F_{B}\right|^{2}\right. \\
& \left.-\left[4 A_{1 L} A_{2 R}{ }^{*}-\left(A_{1 L}-2 A_{2 R}\right)\left(F_{L L}{ }^{*}+F_{L R}{ }^{*}+\frac{1}{2} F_{B}{ }^{*}\right)-\frac{1}{2} F_{L L} F_{B}{ }^{*}+\text { h.c. }\right]\right\}, \quad \text { (A.2) } \\
\Gamma\left(\ell \rightarrow \ell^{\prime} \ell^{\prime \prime} \bar{\ell}^{\prime \prime \prime}\right)_{\text {Type 3 }} & =\frac{\alpha^{2} m_{\ell}^{5}}{192 \pi}\left|F_{B}\right|^{2},
\end{aligned}
$$

where

$$
A_{1 L}=\frac{F_{L}^{\gamma}}{q^{2}}, \quad A_{2 R}=\frac{2 F_{M}^{\gamma}}{m_{\ell}}, \quad F_{L L}=-\frac{g_{L} F_{L}^{Z}}{e m_{Z}^{2}}, \quad F_{L R}=-\frac{g_{R} F_{L}^{Z}}{e m_{Z}^{2}}
$$

\section{References}

[1] R. N. Mohapatra and P. B. Pal, World Sci. Lect. Notes Phys. 60 (1998) 1, [World Sci. Lect. Notes Phys. 72 (2004) 1].

[2] B. Pontecorvo, Sov. Phys. JETP 7, 172 (1958) [Zh. Eksp. Teor. Fiz. 34, 247 (1957)].

[3] Z. Maki, M. Nakagawa and S. Sakata, Prog. Theor. Phys. 28, 870 (1962). doi:10.1143/PTP.28.870

[4] N. Cabibbo, Phys. Rev. Lett. 10, 531 (1963). doi:10.1103/PhysRevLett.10.531

[5] M. Kobayashi and T. Maskawa, Prog. Theor. Phys. 49, 652 (1973). doi:10.1143/PTP.49.652

[6] P. Minkowski, Phys. Lett. 67B, 421 (1977). doi:10.1016/0370-2693(77)90435-X

[7] M. Gell-Mann, P. Ramond and R. Slansky, Conf. Proc. C 790927 (1979) 315 [arXiv:1306.4669 [hep-th]].

[8] R. N. Mohapatra and G. Senjanovic, Phys. Rev. Lett. 44, 912 (1980). doi:10.1103/PhysRevLett.44.912

[9] T. P. Cheng and L. F. Li, “Gauge Theory Of Elementary Particle Physics”, Oxford, Uk: Clarendon (1984) 536 P. (Oxford Science Publications)

[10] S. T. Petcov, Sov. J. Nucl. Phys. 25, 340 (1977) [Yad. Fiz. 25, 641 (1977)] Erratum: [Sov. J. Nucl. Phys. 25, 698 (1977)] Erratum: [Yad. Fiz. 25, 1336 (1977)].

[11] G. Hernández-Tomé, G. López Castro and P. Roig, Eur. Phys. J. C 79, no. 1, 84 (2019) doi:10.1140/epjc/s10052-019-6563-4 [arXiv:1807.06050 [hep-ph]].

[12] R. N. Mohapatra and J. W. F. Valle, Phys. Rev. D 34, 1642 (1986). doi:10.1103/PhysRevD.34.1642

[13] J. Bernabéu, A. Santamaría, J. Vidal, A. Méndez and J. W. F. Valle, Phys. Lett. B 187, 303 (1987). doi:10.1016/0370-2693(87)91100-2

[14] M. Malinsky, J. C. Romao and J. W. F. Valle, Phys. Rev. Lett. 95, 161801 (2005) doi:10.1103/PhysRevLett.95.161801 [hep-ph/0506296]. 
[15] M. Tanabashi et al. [Particle Data Group], Phys. Rev. D 98, no. 3, 030001 (2018). doi:10.1103/PhysRevD.98.030001

[16] E. Kou et al. [Belle-II Collaboration], arXiv:1808.10567 [hep-ex].

[17] R. Aaij et al. [LHCb Collaboration], JHEP 1502, 121 (2015) doi:10.1007/JHEP02(2015)121 [arXiv:1409.8548 [hep-ex]].

[18] A. Ilakovac and A. Pilaftsis, Nucl. Phys. B 437, 491 (1995) doi:10.1016/0550-3213(94)00567-X [hep-ph/9403398].

[19] G. Hernández-Tomé, J. I. Illana, G. López-Castro, M. Masip and P. Roig, Effects of heavy Majorana neutrinos on lepton flavor violating processes, to appear.

[20] J. I. Illana, M. Jack and T. Riemann, hep-ph/0001273.

[21] J. I. Illana and T. Riemann, Phys. Rev. D 63, 053004 (2001).

[22] A. Denner, H. Eck, O. Hahn and J. Kublbeck, Nucl. Phys. B 387, 467 (1992). doi:10.1016/0550-3213(92)90169-C

[23] E. Fernández-Martínez, J. Hernández-García and J. López-Pavon, JHEP 1608, 033 (2016) doi:10.1007/JHEP08(2016)033 [arXiv:1605.08774 [hep-ph]].

[24] A. Abada, M. E. Krauss, W. Porod, F. Staub, A. Vicente and C. Weiland, JHEP 1411, 048 (2014) doi:10.1007/JHEP11(2014)048 [arXiv:1408.0138 [hep-ph]].

[25] A. Abada and A. M. Teixeira, Front. in Phys. 6, 142 (2018) doi:10.3389/fphy.2018.00142 [arXiv:1812.08062 [hep-ph]]. 